\title{
Regulatory effects of chicken TRIM25 on the replication of ALV-A and the MDA5-mediated type I interferon response
}

\author{
Jin-run Zhou ${ }^{1,2+}$, Jun-hong Liu ${ }^{1,2+}$, Hong-mei Li ${ }^{1,2 \dagger}$, Yue Zhao ${ }^{1,2}$, Ziqiang Cheng ${ }^{1,2}$, Yan-meng Hou ${ }^{1,2}$
} and Hui-jun Guo ${ }^{1,2^{*}}$ (D)

\begin{abstract}
This study focuses on the immunoregulatory effects of chicken TRIM25 on the replication of subgroup A of avian leukosis virus (ALV-A) and the MDA5-mediated type I interferon response. The ALV-A-SDAU09C1 strain was inoculated into DF1 cells and 1-day-old SPF chickens, and the expression of TRIM25 was detected at different time points after inoculation. A recombinant overexpression plasmid containing the chicken TRIM25 gene (TRIM25-GFP) was constructed and transfected into DF1 cells to analyse the effects of the overexpression of chicken TRIM25 on the replication of ALV-A and the expression of MDA5, MAVS and IFN- $\beta$. A small interfering RNA targeting chicken TRIM25 (TRIM25-siRNA) was prepared and transfected into DF1 cells to assess the effects of the knockdown of chicken TRIM25 on the replication of ALV-A and the expression of MDA5, MAVS and IFN- $\beta$. The results showed that chicken TRIM25 was significantly upregulated at all time points both in ALV-A-infected cells and in ALV-A-infected chickens. Overexpression of chicken TRIM25 in DF1 cells dramatically decreased the antigenic titres of ALV-A in the cell supernatant and upregulated the relative expression of MDA5, MAVS and IFN- $\beta$ induced by ALV-A or by poly(l:C); in contrast, knockdown of chicken TRIM25 significantly increased the antigenic titres of ALV-A and downregulated the relative expression of MDA5, MAVS and IFN- $\beta$. It can be concluded that chicken TRIM25 can inhibit the replication of ALV-A and upregulate the MDA5 receptor-mediated type I interferon response in chickens. This study can help improve the understanding of the antiviral activities of chicken TRIM25 and enrich the knowledge of antiviral responses in chickens.
\end{abstract}

Keywords: chicken TRIM25, subgroup A of avian leukosis virus, antiviral bioactivities, type I interferon response

\section{Introduction}

Tripartite motif 25 (TRIM25) is a tripartite motif (TRIM) protein family member and is characterized by a conserved structural domain at the $\mathrm{N}$ terminus, including a

*Correspondence: hjguo@sdau.edu.cn

†Jin-run Zhou, Jun-hong Liu and Hong-mei Li contributed equally to this work

${ }^{1}$ Shandong Provincial Key Laboratory of Animal Biotechnology and Disease Control and Prevention, Tai'an 271018, China

Full list of author information is available at the end of the article catalytic RING domain, two B-box domains, and a coiledcoil dimerization domain $[1,2]$. In addition, TRIM25 has a C-terminal SPRY domain that binds to target proteins, such as the RIG-I receptor [3, 4].

TRIM25 was first reported in 1993 as an oestrogenresponsive finger protein (EFP) that regulates the oestrogen response [5]. Later, it was also found that the protein can mediate RNA virus infection and induce the antiviral response by ubiquitinating intracellular effectors $[6$, 7]. TRIM25 has been confirmed to be involved not only

(c) The Author(s) 2020. This article is licensed under a Creative Commons Attribution 4.0 International License, which permits use, sharing, adaptation, distribution and reproduction in any medium or format, as long as you give appropriate credit to the original author(s) and the source, provide a link to the Creative Commons licence, and indicate if changes were made. The images or other third party material in this article are included in the article's Creative Commons licence, unless indicated otherwise in a credit line to the material. If material is not included in the article's Creative Commons licence and your intended use is not permitted by statutory regulation or exceeds the permitted use, you will need to obtain permission directly from the copyright holder. To view a copy of this licence, visit http://creativeco mmons.org/licenses/by/4.0/. The Creative Commons Public Domain Dedication waiver (http://creativecommons.org/publicdomain/ zero/1.0/) applies to the data made available in this article, unless otherwise stated in a credit line to the data. 
in the immune response against many RNA viruses [8] but also in the occurrence of tumours in humans, as an important innate immune molecule [9]. It is becoming clear that TRIM25 has a dual role in the regulation of type I interferons (IFNs), such as IFN- $\alpha / \beta$; that is, TRIM25 positively regulates IFNs by the K63-linked ubiquitination of RIG-I and negatively regulates IFNs by the K48-linked ubiquitination of MAVS, and it can thus inhibit or enhance the replication of some RNA viruses $[10,11]$.

In recent years, many animal TRIM25 genes have been cloned, and their molecular structures and antiviral effects have been reported [12, 13]. The chicken TRIM25 gene was cloned in 2015, and it was confirmed that its protein was widely expressed in chickens, especially in immune organs $[14,15]$. However, due to the absence of RIG-I in chickens [16, 17], little is known about the immunoregulatory effects of chicken TRIM25 on the replication of RNA viruses and type I IFN responses. In 2015, it was reported that stimulator of IFN genes (STING), as an important regulator of chicken innate immune signalling, might be involved in the MDA5 signalling pathway in chicken cells [18]. In this study, it was found that chicken TRIM25 can inhibit the replication of subgroup A avian leukosis virus (ALV-A) and upregulate the MDA5-mediated IFN- $\beta$ response in chicken cells.

\section{Materials and methods}

\section{Cells, virus strain and reagents}

DF1 cells, a chicken embryonic fibroblast cell line in which exogenous ALV can replicate, were a gift from Professor Zhizhong Cui. The polyclonal antibody (PAb) against chicken TRIM25 was prepared by our laboratory [15]. The ALV-A-SDAU09C1 strain was isolated from imported meat-type grandparent chickens in 2009 and stored at $-80{ }^{\circ} \mathrm{C}$ until use [19]. The $50 \%$ tissue culture infective dose $\left(\mathrm{TCID}_{50}\right)$ of the virus was determined using a limiting dilution assay in a 96-well plate covered with DF1 cells according to the Reed-Muench method. The positive cells were identified using an indirect immunofluorescence assay (IFA) mediated by a monoclonal antibody (MAb) against ALV-A. The pEGFP-C1 vector containing the green fluorescent protein gene was purchased from Beijing Tiangen Biotechnology Co., Ltd., and a small interfering RNA (siRNA) was synthesized by Shanghai GenePharma Biotechnology Co., Ltd.

Polyinosinic-polycytidylic acid (poly(I:C)) sodium salt (number: P1530) was purchased from Sigma, dissolved at $5 \mathrm{mg} / \mathrm{mL}$ with sterile PBS and stored at $-20{ }^{\circ} \mathrm{C}$. Before transfection, poly(I:C) was diluted to $1 \mathrm{mg} / \mathrm{mL}$ and mixed with Lipofectamine 3000 reagent in a $200 \mu \mathrm{L}$ reaction system with Opti-MEM according to the instructions.
Finally, transfection medium containing $2 \mu \mathrm{g} / \mathrm{mL}$ poly(I:C) was added to prepared DF1 cells.

\section{Viral infection}

In vitro, DF1 cells were inoculated with $10^{2} \mathrm{TCID}_{50}$ of the ALV-A-SDAU09C1 strain, and the inoculum was discarded at $2 \mathrm{~h}$ post inoculation (hpi); fresh maintenance medium was added onto the infected cells. The cell samples were collected at $0,2,6,12,24,72,120$ and 168 hpi. RNA was extracted from the cells to detect the mRNA levels of chicken TRIM25 by real-time PCR (RT-PCR). In addition, the total proteins were also extracted from the cells to detect the protein levels of chicken TRIM25 by western blot mediated with the PAb against chicken TRIM25.

In vivo, thirty 1-day-old SPF chickens (White Leghorn) were intraperitoneally inoculated with $10^{4} \mathrm{TCID}_{50}$ of the ALV-A-SDAU09C1 strain as the ALV-A infected group; in addition, thirty 1-day-old SPF chickens were intraperitoneally inoculated with DMEM as the control group. A total of $0.5 \mathrm{~mL}$ of solution was injected into each chicken. Blood samples were collected from all the experimental chickens, and the antigenic titres of ALV-A in the plasma samples were detected by ELISA. Six chickens per group were randomly killed at 1, 2, 3, and 4 weeks post inoculation (wpi), and RNA was extracted from the spleen, liver, kidney and lung of the sacrificed chickens to detect the expression of chicken TRIM25 mRNA by RT-PCR.

\section{Amplification of the chicken TRIM25 gene}

The chicken TRIM25 gene containing the open-reading frame (ORF) was amplified from the spleen of chickens according to a published method [15]. The primers (forward: 5'-CCCGAATTCTTGCAACAACCAGGA AACG-3'; reverse: $5^{\prime}$-CCCGCGGCCGCTCAGTGATGA TGATGATGATGCCTGCTACGGCGGTGACCC-3') were designed according to the chicken TRIM25 gene sequence (NM_001318458.1) published in GenBank and were synthesized by Liuhe Huada Gene Biological Limited Co. Fresh spleen tissues were collected from HyLine Brown chickens, RNA was extracted using an RNA Extraction Kit, and reverse transcription was carried out to obtain cDNA encoding chicken TRIM25. The target gene was amplified from the obtained cDNA by polymerase chain reaction $(\mathrm{PCR})$ under the following conditions: initial denaturation at $95^{\circ} \mathrm{C}$ for $3 \mathrm{~min} ; 30$ cycles of denaturation at $95^{\circ} \mathrm{C}$ for $30 \mathrm{~s}$, annealing at $61^{\circ} \mathrm{C}$ for $60 \mathrm{~s}$, and extension at $72{ }^{\circ} \mathrm{C}$ for $72 \mathrm{~s}$; and a final extension at $72{ }^{\circ} \mathrm{C}$ for $10 \mathrm{~min}$. The PCR products were analysed using $1 \%$ agarose gel electrophoresis, and then, the PCR products that were recovered using a DNA Gel Recovery Kit were sequenced by Shanghai Biological Engineering Technology Services Limited Co. 


\section{Knockdown of chicken TRIM25 by siRNA}

A small interfering RNA (siRNA) targeting chicken TRIM25, named TRIM25-siRNA in Table 1, was designed and synthesized. DF1 cells were seeded at $5 \times 10^{5}$ cells/ well in 6-well plates and transfected with $50 \mathrm{nM}$ TRIM25siRNA mixed with $2 \mu \mathrm{L}$ of Lipofectamine 3000 (Invitrogen) according to the manufacturer's instructions. The knockdown effect of TRIM25-siRNA on TRIM25 in DF1 cells was evaluated at $24 \mathrm{~h}$ post transfection by real-time RTPCR and by western blot conducted with the PAb against chicken TRIM25, and the efficiency was determined as the ratio of TRIM25 expression in the knockdown samples relative to that in the negative control samples.

\section{Construction of an overexpression plasmid containing chicken TRIM25}

After the chicken TRIM25 gene containing the complete ORF fragment with a size of 1881 bp was successfully amplified, the recombinant overexpression plasmid named TRIM25-GFP was constructed using the cloning vector pMD18-T and the fluorescent expression vector pEGFPC1. The TRIM25-GFP plasmid and blank GFP plasmid were transfected into DF1 cells with $2 \mu \mathrm{L}$ of Lipofectamine 3000 (Invitrogen) according to the manufacturer's instructions, and the expression efficiency of the TRIM25-GFP plasmid in DF1 cells was evaluated by the real-time RTPCR method and Western blot mediated with the PAb against chicken TRIM25 at $24 \mathrm{~h}$ post transfection; simultaneously, the fluorescence was observed using fluorescence microscopy.

\section{Overexpression and knockdown of chicken TRIM25 regulation of ALV-A replication}

The TRIM25-GFP plasmid and TRIM25-siRNA were transfected into DF1 cells; the GFP plasmid and NCsiRNA were used as controls. At $4 \mathrm{~h}$ post transfection, the DF1 cells were inoculated with $10^{2} \mathrm{TCID}_{50}$ of the ALVSDAU09C1 strain; the inoculum was discarded, and new culture medium was added to the cells, which were then cultured for 7 days at $37^{\circ} \mathrm{C}$. Cell supernatant samples were collected at 6 hpi, 12 hpi, 24 hpi, 48 hpi, 96 hpi, and 144 hpi, and the antigenic titres of ALV-A were detected by ELISA using an IDEXX ALV P27 Antigen Kit (IDEXX USA Inc., Beijing, China). Each sample was tested in triplicate.

\section{Overexpression and knockdown of chicken TRIM25 regulation of IFN- $\beta$ responses}

The TRIM25-GFP plasmid, TRIM25-siRNA, GFP plasmid and NC-siRNA were transfected into DF1 cells with Lipofectamine 3000 reagent. After $4 \mathrm{~h}, 2 \mu \mathrm{g} / \mathrm{mL}$ poly(I:C) was transfected or $10^{2} \mathrm{TCID}_{50}$ of the ALV-SDAU09C1 strain was inoculated into DF1 cells to activate type I interferon responses according to the published method [18]. Cell samples were collected at $120 \mathrm{~h}$ (5 days) post inoculation (hpi). The mRNA transcript levels of chicken TRIM25, MDA5, MAVS, IFN- $\beta$ and GAPDH in the cells were detected by real-time RT-PCR, and their relative expression levels were calculated. Each sample was tested in triplicate.

\section{Detection of ALV-A antigenic titres by ELISA}

To determine the titres of ALV-A in the cell supernatant samples or in the plasma samples, the P27 antigen of ALV-A was detected using an ALV P27 antigen ELISA kit (IDEXX USA Inc., Beijing, China) as described [20]. In brief, $1 \mathrm{~mL}$ of the dilution fluid from the kit was added to the samples, and the samples were subjected to three freeze-thaw cycles. After sedimentation, the supernatants of the samples were evaluated for ALV P27 antigens in accordance with the manufacturer's protocol. The antigenic titres of ALV-A were determined by calculating the sample-to-positive control $(\mathrm{S} / \mathrm{P})$ ratio using the formula [(mean of sample optical density)-(mean of negative control optical density)]/[(mean of positive control optical density)-(mean of negative control optical density)]. Each sample was tested in triplicate.

\section{IFA for detecting ALV-A in DF-1 cells}

The TRIM25-GFP plasmid and TRIM25-siRNA were transfected into DF1 cells, and the GFP plasmid and NCsiRNA were used as negative controls. Fresh medium was added to DF1 cells at $6 \mathrm{~h}$ post transfection to maintain the culture for $24 \mathrm{~h}$; then, $10^{2} \mathrm{TCID}_{50}$ of the ALV-A strain was inoculated into DF1 cells, the inoculum was discarded at $2 \mathrm{hpi}$, and DF1 cells were cultured with $1 \%$ serum medium for $72 \mathrm{~h}$, washed with PBS and fixed with 4\% paraformaldehyde for $20 \mathrm{~min}$. Anti-ALV-A antibody (1:100) was incubated for $1 \mathrm{~h}, \mathrm{CY} 3$-labelled secondary antibody (1:1000, Beyotime) was incubated at $37^{\circ} \mathrm{C}$ for $1 \mathrm{~h}$, and the cells were washed with PBS, then incubated

Table 1 TRIM25-SiRNA and control SiRNA sequences used in this study

\begin{tabular}{llll}
\hline Purpose & Name & Sequence $\left(\mathbf{5}^{\prime}\right.$ to3') & Accession no \\
\hline Knockdown & TRIM25-SiRNA & GCUAACGUCACGCUGGAUUTT & NM_001318458.1 \\
Control & NC-SiRNA & UUCUCCGAACGUGUCACGUTT & \\
\hline
\end{tabular}


with DAPI for $5 \mathrm{~min}$, and observed under a fluorescence microscope after washing with PBS.

\section{Real-time RT-PCR}

Real-time RT-PCR using SYBR Green was performed on a Roche LightCycler 96 to detect the transcript levels of chicken TRIM25, MDA5, MAVS, IFN- $\beta$ and GAPDH using a Rotor-Gene SYBR Green PCR Kit (QIAGEN, Valencia, CA) and the corresponding primers listed in Table 2 . The reaction mixture, which contained $2 \times$ UltraSYBR Mixture, $100 \mathrm{nM}$ forward primer, $100 \mathrm{nM}$ reverse primer and $1 \mu \mathrm{L}$ of cDNA, was subjected to the following thermal cycling conditions: 1 cycle of predenaturation at $95{ }^{\circ} \mathrm{C}$ for $10 \mathrm{~min}$; 40 cycles of amplification at $95^{\circ} \mathrm{C}$ for $15 \mathrm{~s}$ and $60^{\circ} \mathrm{C}$ for $1 \mathrm{~min}$; and 1 cycle of melting at $95^{\circ} \mathrm{C}$ for $15,60^{\circ} \mathrm{C}$ for $1 \mathrm{~min}$ and $95^{\circ} \mathrm{C}$ for $15 \mathrm{~s}$.

\section{Statistics analysis}

All the data are expressed as the mean \pm standard deviation. The intergroup differences were analysed with ANOVA software, followed by Student-Newman-Keuls tests of multiple comparisons. $\mathrm{P}<0.05$ was considered statistically significant. $\mathrm{P}<0.01$ was considered highly significant.

\section{Results}

TRIM25 expression in DF1 cells is dramatically upregulated by ALV-A infection

As shown in Figure 1A, the mRNA level of chicken TRIM25 in DF1 cells was significantly upregulated from 2 to $168 \mathrm{hpi}$ and reached the highest point at $120 \mathrm{hpi}$. The western blot results in Figure 1B further confirmed this finding. These results suggest that chicken TRIM25 might be involved in the cell responses to ALV-A infection.

\section{TRIM25 expression in chickens is significantly upregulated} by ALV-A infection

To further confirm the expression of TRIM25 after ALV-A infection in vivo, 1-day-old SPF chickens were infected with the ALV-A strain, and the mRNA expression of TRIM25 in five organs, the spleen, liver, bursa, lung and kidney, was detected at $7 \mathrm{dpi}, 14 \mathrm{dpi}, 21 \mathrm{dpi}$ and
28 dpi. The results in Figure 2A-D show that the relative mRNA expression of TRIM25 in these five organs of the infected chickens was increased by different ratios compared with that in these five organs of the control chickens, especially in the spleens and livers, as the relative expression was increased by 4 to 10 -fold in the spleens and by 3.5 to 6 -fold in the livers. Simultaneously, it was also found that the expression of TRIM25 decreased with increasing ALV-A inoculation time from $7 \mathrm{dpi}$ (Figure $2 \mathrm{~A}$ ) to $28 \mathrm{dpi}$ (Figure 2D); however, the ALV-A antigenic titres in the infected chickens' blood were clearly increased from 7 to $28 \mathrm{dpi}$ (Figure 2E). These results suggest that TRIM25 is involved in the immune responses against ALV-A infection in chickens and may negatively regulate the replication of ALV-A in chickens.

\section{Overexpression of chicken TRIM25 can inhibit the replication of ALV-A}

To analyse the effects of chicken TRIM25 on the replication of ALV-A, a new recombinant plasmid expressing the chicken TRIM25 ORF fragment gene (TRIM25-GFP) was designed and constructed with the fluorescent expression vector $\mathrm{pEGFP-C1}$. The results show that the chicken TRIM25 gene containing a complete ORF fragment with a size of 1881 bp was successfully amplified, and its cloning vector (pMD18-T) and expression vector containing the EGFP gene (TRIM25-GFP) were also successfully prepared (data not shown). The TRIM25-GFP plasmid and GFP plasmid were transfected into DF-1 cells at a concentration of $1 \mu \mathrm{g} / \mathrm{mL}$, and green fluorescence specifically occurred in the transfected cells at $24 \mathrm{~h}$ post transfection (hpt) and was clearly observed with fluorescence microscopy at $72 \mathrm{hpt}$ (data not shown). The mRNA and protein expression levels of chicken TRIM25 were detected by QPCR and western blot, and the results showed that TRIM25 mRNA levels in the TRIM25-GFPtransfected cells were significantly higher than those in the GFP-transfected cells and in the blank control cells (Figure 3A). The western blotting results further confirmed this finding (Figure 3B).

After inoculation with the ALV-A-SDAU09C1 strain, DF1 cells were examined for the presence of ALV-A using

Table 2 Sequences of the primers used in real-time RT-PCR reaction

\begin{tabular}{lll}
\hline Primer name & Sequence $\left(\mathbf{5}^{\prime}\right.$ to $\mathbf{3}^{\prime} \mathbf{)}$ & \\
\cline { 2 - 3 } & Forward & Reverse \\
\hline GAPDH & GGTGGTGCTAAGCGTGTTA & CCCTCCACAATGCCAA \\
TRIM25 & TACAACCACCACCCTCA & GATGCCAATGCCACAG \\
MDA5 & TGAAAGCCTTGCAGATGACTTA & GCTGTTTCAAATCCTCCGTTAC \\
MAVS & CACCCACGAGGTCATGTG & TGCTTCATCTGGGACATCATTG \\
IFN- $\beta$ & TCCAGCTCCTTCAGAATACG & TGCGGTCAATCCAGTGTT \\
\hline
\end{tabular}


A

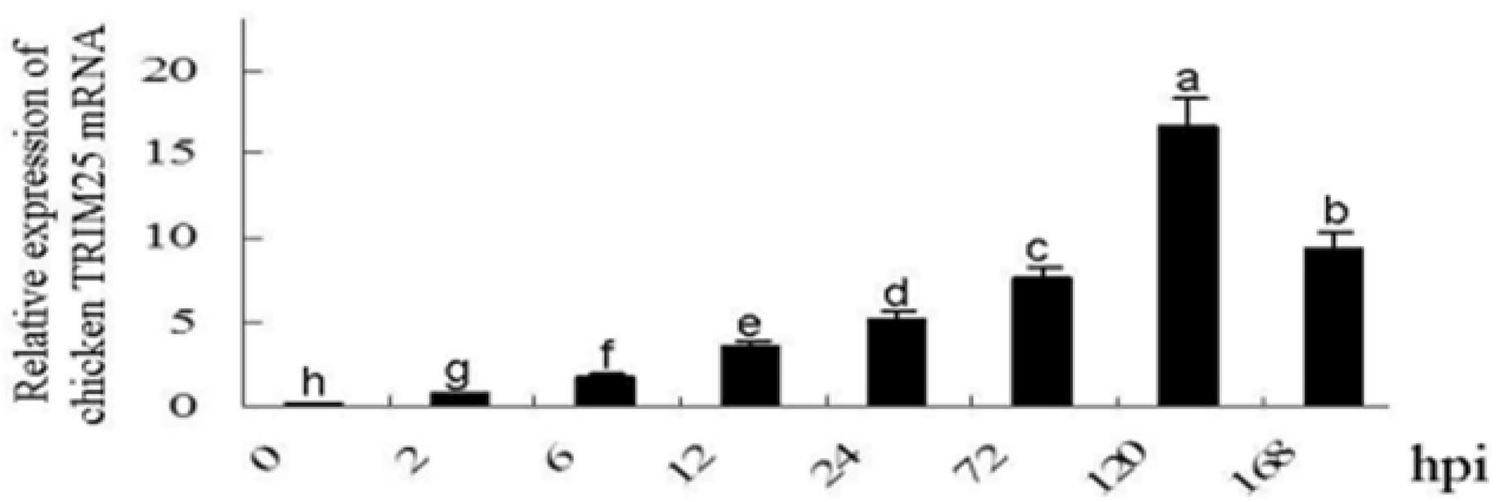

B

$\begin{array}{llllllll}0 & 2 & 6 & 12 & 24 & 72 & 120 & 168\end{array}$

TRIM25

GAPDH

Figure 1 The mRNA relative expression (A) and the protein expression (B) of chicken TRIM25 in the DF1 cells at different time points after infection with the ALV-A-SDAU09C1 strain. Different letters on the bars indicate statistically significant differences between the groups $(P<0.05)$; hpi, hours post inoculation.
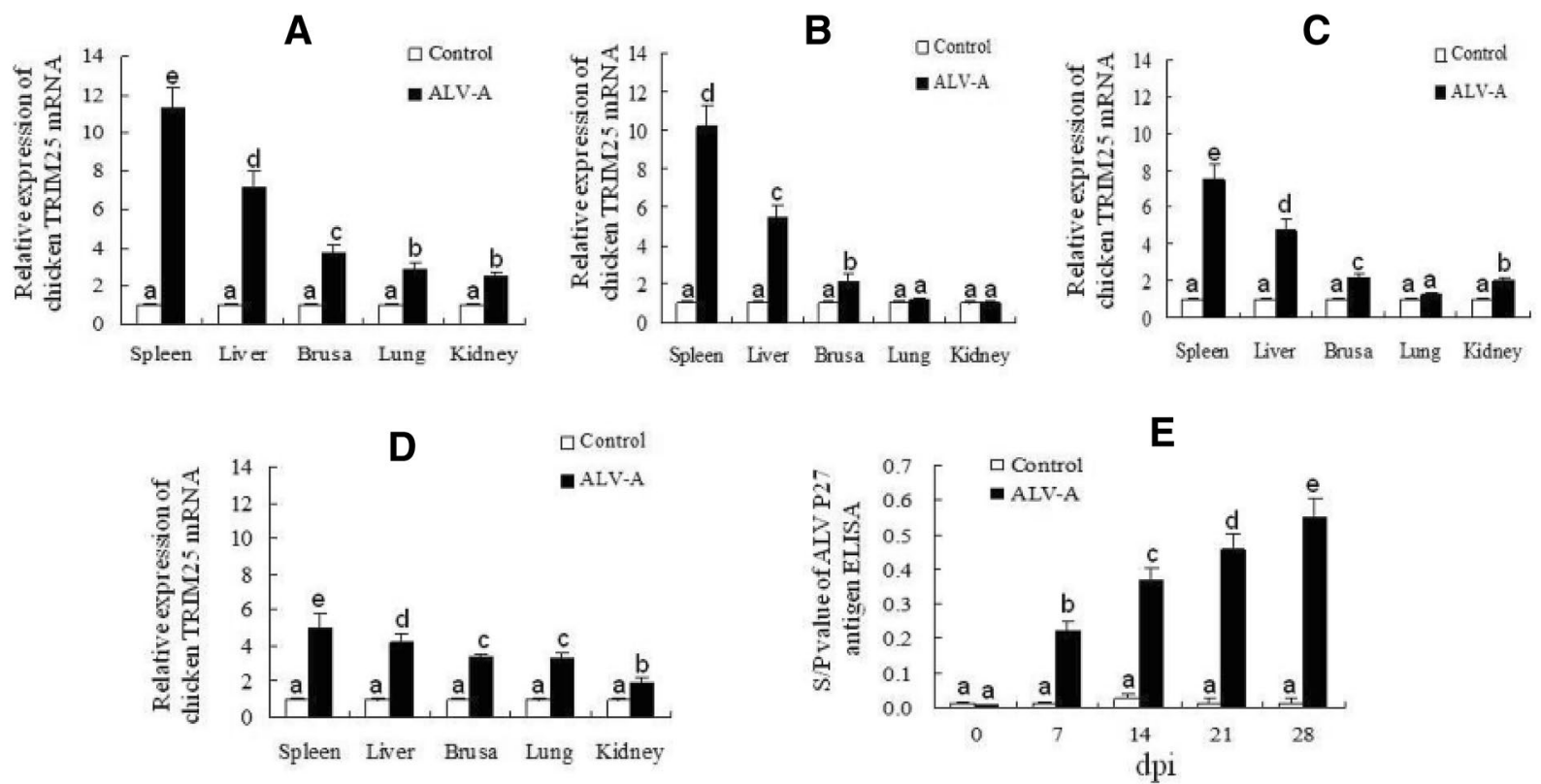

Figure 2 The relative expression of TRIM25 mRNA in spleen, liver, bursa, lung and kidney of chickens at 7 dpi (A), 14 dpi (B), 21 dpi (C), 28 dpi (D) and the antigen titers of ALV-A in the blood (E). Different letters on the bars indicate statistically significant differences $(P<0.05)$ and the same letter indicates no significant difference $(P>0.05)$ between the groups; dpi, days post inoculation. 

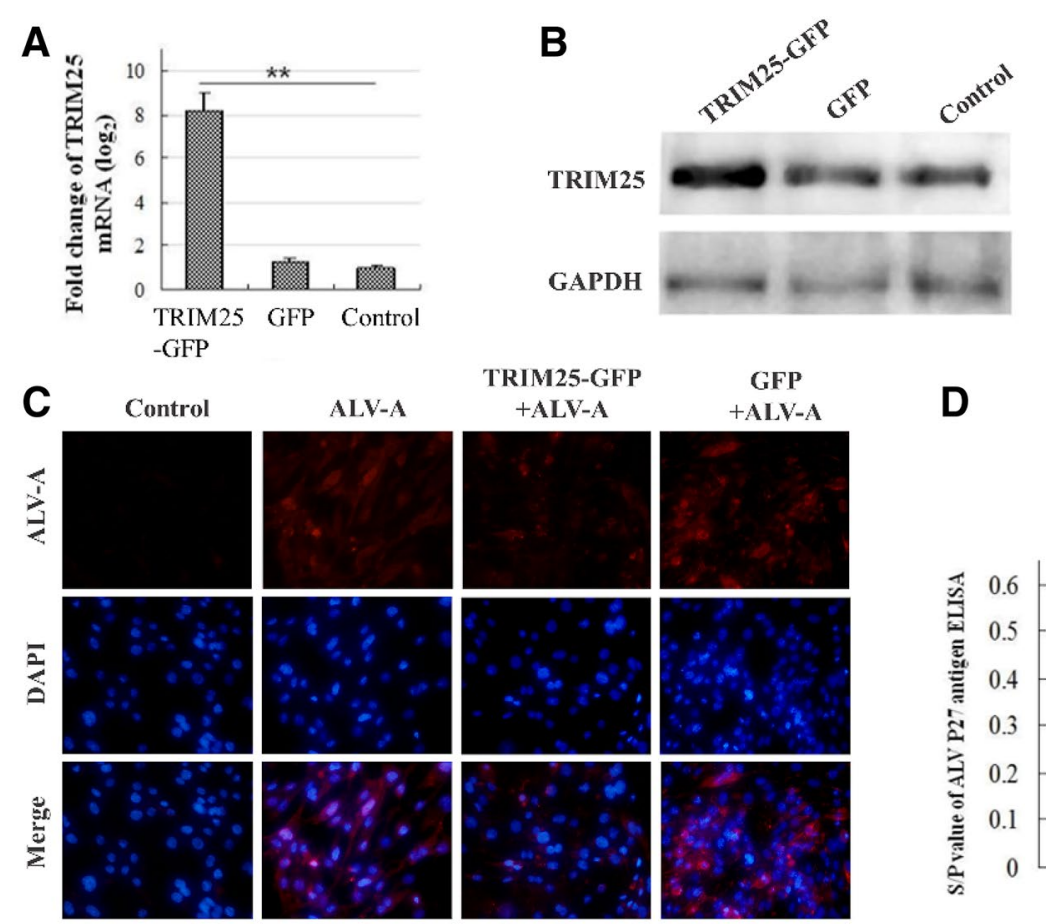

D
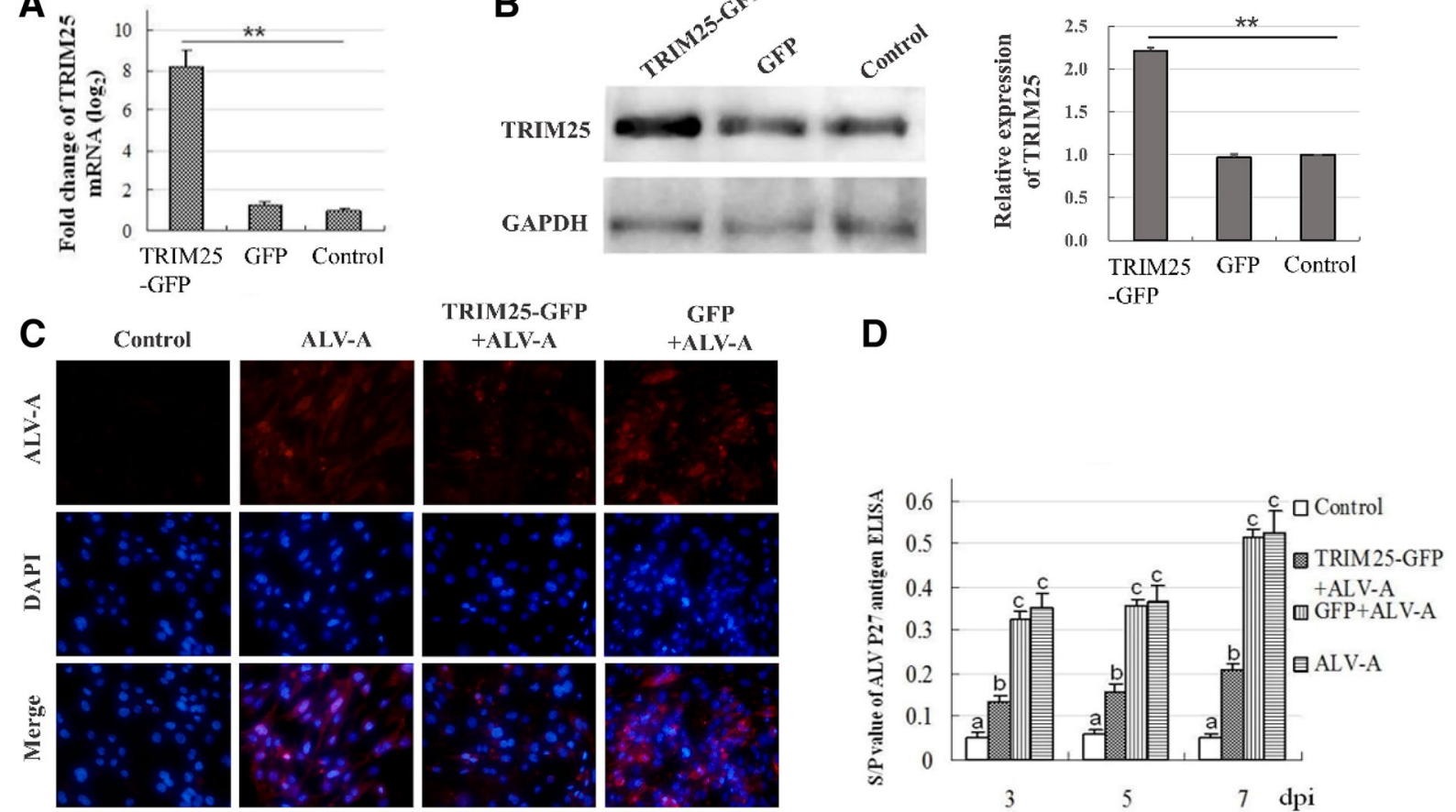

Figure 3 Overexpression of chicken TRIM25 can inhibit the replication of ALV-A. The expression of chicken TRIM25 in the DF1 cells transfected with the TRIM25-GFP plasmid and GFP plasmid were detected by QPCR (A) and western blot (B) (the grey signals were analyzed using Image I software and the relative ratios were statistically calculated, ${ }^{* *}, \mathrm{P}<0.01$ ). The intracellular proliferation of ALV-A after TRIM25-GFP transfection was detected by immunofluorescence at $3 \mathrm{dpi}(\mathbf{C})$. The effects of TRIM25-GFP on the antigen titers of ALV-A in DF1 cells at different days post inoculation with the ALV-A-SDAU09C1 strain were detected by ELISA (D). Different letters on the bars indicate statistically significant differences $(P<0.05)$ and the same letter indicates no significant difference $(P>0.05)$ between the groups; dpi, days post inoculation.

IFA at 3 dpi. The results showed that ALV-A levels in the TRIM25-GFP-transfected cells were significantly lower than those in the GFP transfected cells and ALV-A positive control cells (Figure 3C). The antigen titres of ALV-A in DF1 cell supernatants were detected by ELISA at 3, 5 and 7 dpi. The results in Figure 3D show that the ALV-A titres in the TRIM25-GFP-transfected cells were significantly lower than those in the GFP-transfected cells and those in the ALV-A positive control cells at all time points; furthermore, the decrease ratios were more than $50 \%$, although they were significantly higher than those in the uninfected cells. These results suggest that overexpression of chicken TRIM25 in DF1 cells can clearly inhibit the replication of ALV-A.

\section{Knockdown of chicken TRIM25 can promote the replication of ALV-A}

Based on the principle of siRNA, an siRNA construct targeting chicken TRIM25, named TRIM25-siRNA, and a negative control siRNA construct, named NC-siRNA, were designed, as shown in Table 1 . These plasmids were transfected into DF1 cells at a dose of $50 \mathrm{nM}$, and the expression of TRIM25 mRNA and protein was detected by QPCR at $24 \mathrm{hpt}$ and by western blot at $72 \mathrm{hpt}$, respectively. The results show that TRIM25 mRNA levels in the TRIM25-siRNA-transfected cells were significantly decreased compared with those in the NC-siRNA-transfected cells and in the control cells (Figure 4A). The western blotting results also showed that TRIM25 protein expression in the TRIM25-siRNA-transfected cells was significantly lower than that in the NC-siRNA-transfected cells and that in the control cells (Figure 4B). These results suggest that the designed siRNA can significantly decrease the expression of chicken TRIM25 in DF1 cells and that the knockdown efficiency can reach more than $60 \%$.

After inoculation with the ALV-A-SDAU09C1 strain, ALV-A in DF1 cells was detected using IFA at $3 \mathrm{dpi}$. The results showed that ALV-A levels in the TRIM25siRNA transfected cells were significantly higher than those in the NC-siRNA transfected cells and those in the ALV-A positive control cells (Figure $4 \mathrm{C}$ ). The results in Figure 4D show that the antigen titres of ALV-A in the TRIM25-siRNA-transfected cells were significantly higher than those in the NC-siRNA-transfected cells and those in the ALV-A positive control cells at 3, 5, and 


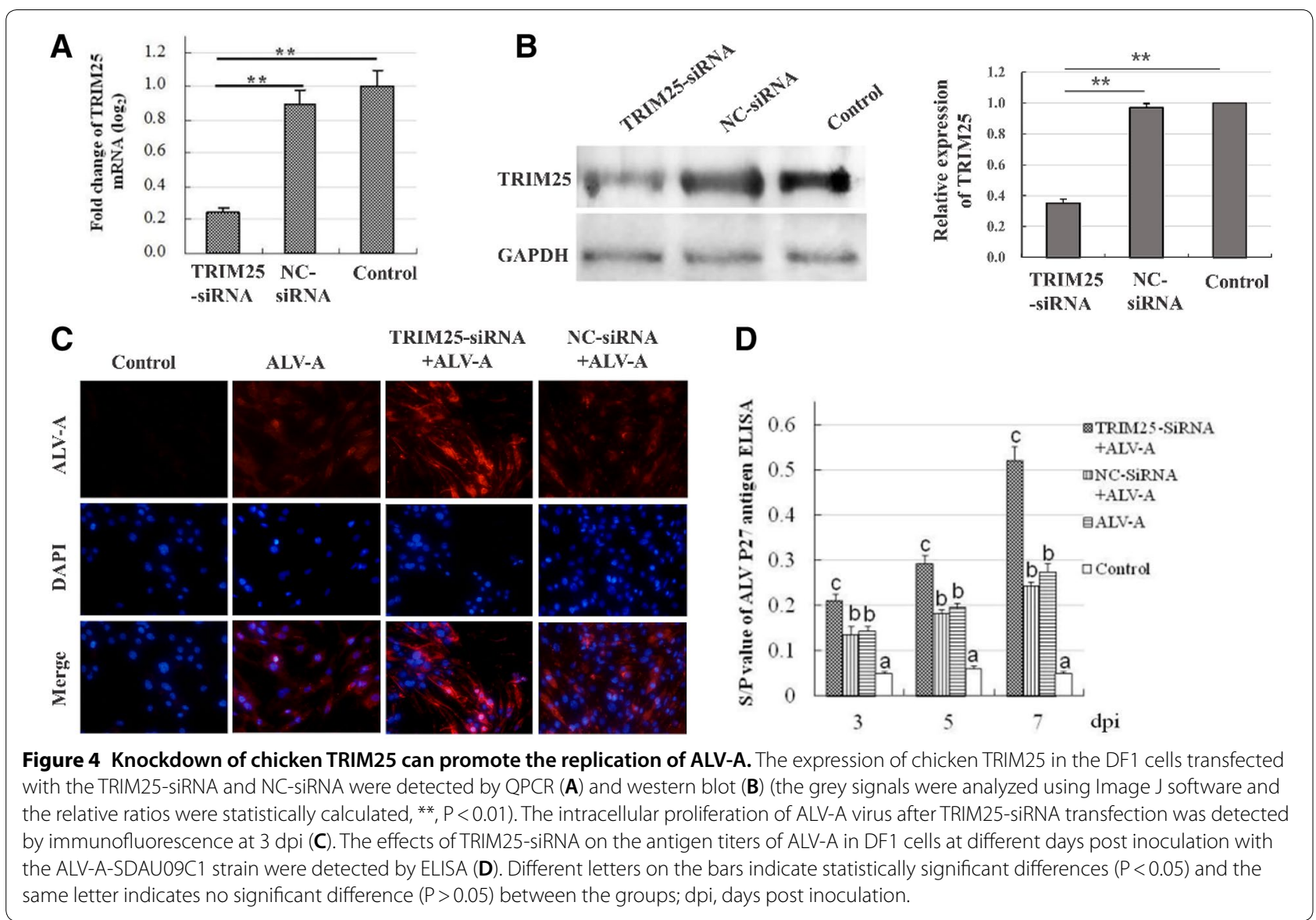

7 days post-inoculation; furthermore, the increase ratios reached $40 \% \sim 104 \%$. These results suggest that knockdown of chicken TRIM25 in DF1 cells can promote the replication of $A L V-A$.

\section{Overexpression of chicken TRIM25 can upregulate the IFN- $\beta$ response induced by ALV-A infection, but knockdown of chicken TRIM25 can downregulate this response}

To analyse the effects of chicken TRIM25 on the type I interferon response induced by ALV-A infection, the TRIM25-GFP plasmid and TRIM25-siRNA were transfected into DF1 cells, and the cells were inoculated with the ALV-A-SDAU09C1 strain. The relative expression levels of TRIM25, MDA5, MAVS and IFN- $\beta$ were detected at $5 \mathrm{dpi}$. The results in Figure 5 show that the mRNA expression of TRIM25 in the TRIM25-GFPtransfected cells was greatly increased and that the expression of MDA5, MAVS and IFN- $\beta$ was also significantly increased after ALV-A infection compared with that after mock infection (Figure 5A). In contrast, the mrna level of TRIM25 in the TRIM25-siRNAtransfected cells was decreased, and the expression of
MDA5, MAVS and IFN- $\beta$ was significantly decreased after ALV-A infection (Figure 5B). These results suggest that chicken TRIM25 can upregulate the IFN- $\beta$ response induced by ALV-A infection.

\section{Chicken TRIM25 can upregulate the MDA-mediated type I interferon response induced by poly $(\mathrm{I}: \mathrm{C})$}

To further elucidate the signalling mechanism by which chicken TRIM25 affects the IFN- $\beta$ response induced by ALV-A infection, poly(I:C), a simplified model of ALV-A strain infection, was co-transfected with the TRIM25-GFP plasmid or TRIM25-siRNA into DF1 cells, and the expression of TRIM25, MDA5, MAVS and IFN- $\beta$ was detected. The results in Figure 6 show that the relative mRNA expression of these molecules was greatly increased in the TRIM25-GFP-transfected cells (Figure 6A) and dramatically decreased in the TRIM25-siRNA-transfected cells (Figure 6B) compared with that in the respective control cells. These results suggest that chicken TRIM25 can also upregulate the MDA5-mediated type I interferon response induced by poly(I:C). 

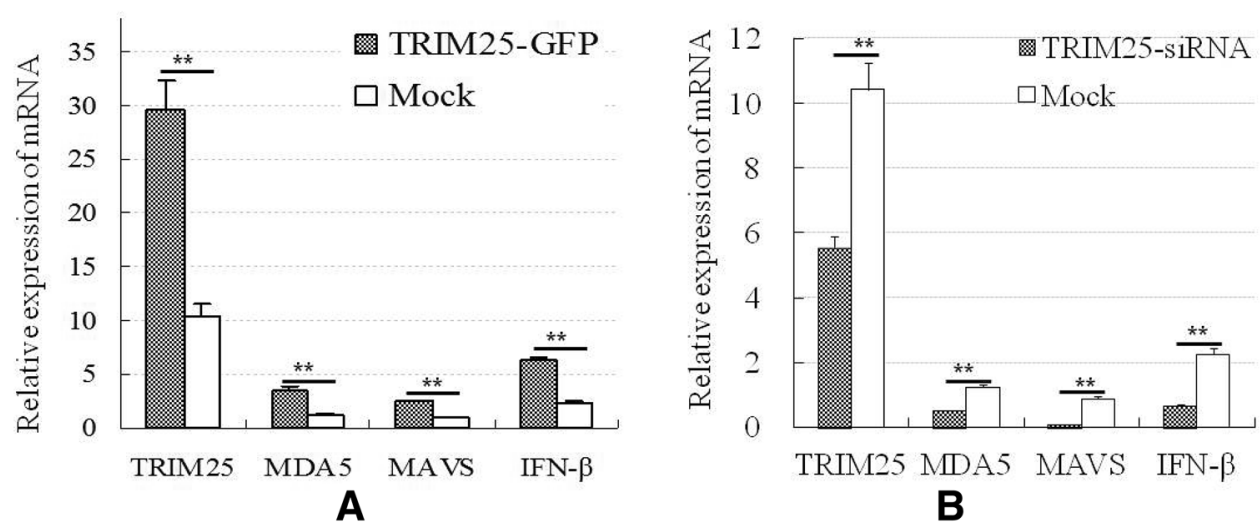

Figure 5 Effects of overexpression (A) and knockdown (B) of chicken TRIM25 on the mRNA transcript levels of MDA5, MAVS and IFN- $\beta$ induced by ALV-A infection in DF1 cells $\left(*, \mathbf{P}<\mathbf{0 . 0 5} ;{ }^{* *}, \mathbf{P}<\mathbf{0 . 0 1}\right)$. The TRIM25-GFP plasmid, TRIM25-siRNA, GFP plasmid (Mock) and NC-siRNA (Mock) were transfected into DF1 cells, respectively; and $10^{2} \mathrm{TCID}_{50}$ of the ALV-SDAU09C1 strain was inoculated at $4 \mathrm{~h}$ post transfection, then the cell samples were collected for detection at $120 \mathrm{~h}$ post inoculation.

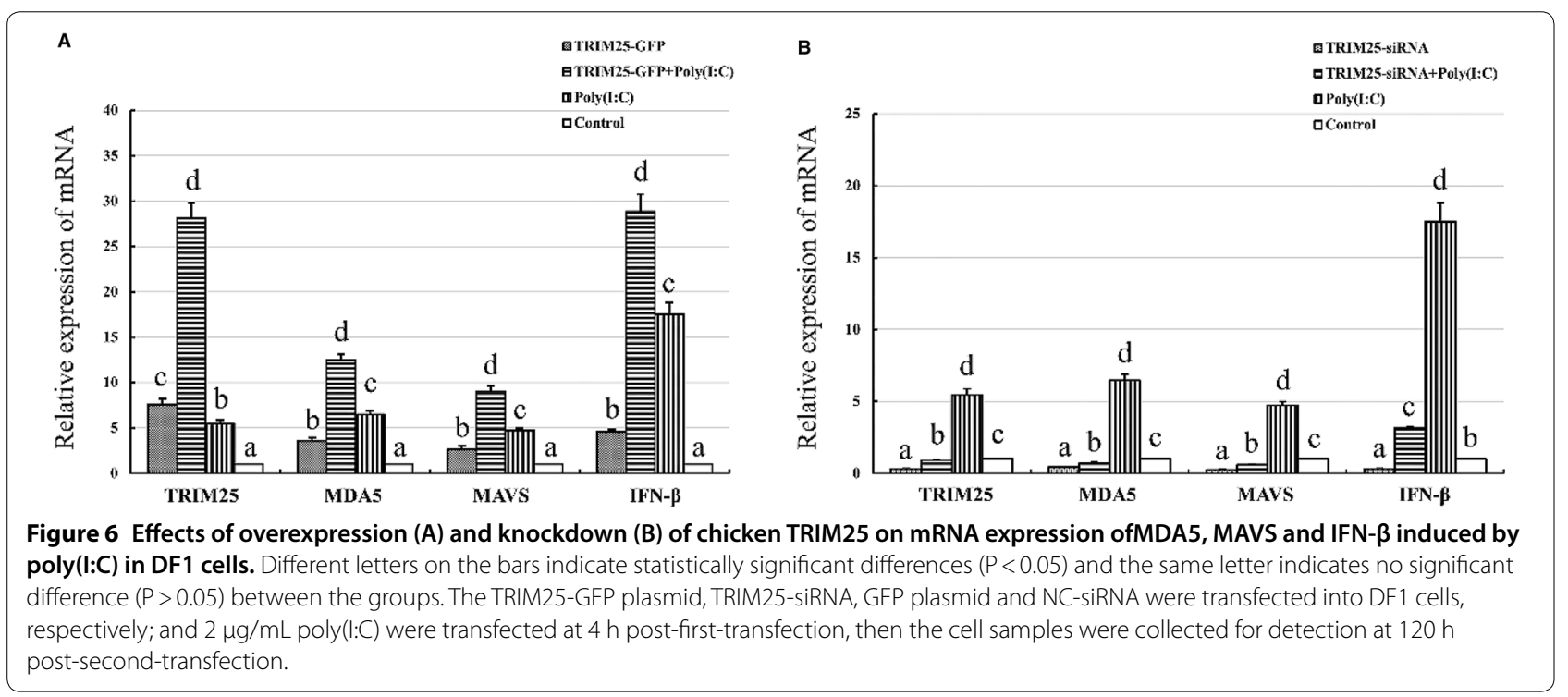

\section{Discussion}

ALV, an oncogenic retrovirus, can cause immunosuppression, decreased production performance, tumours and even death in chickens [20-23]. ALV is divided into 11 subgroups from $\mathrm{A}$ to $\mathrm{K}$ according to the host range, antibody neutralization and receptor interference studies, and seven of these subgroups, namely, ALV-A/ $\mathrm{B} / \mathrm{C} / \mathrm{D} / \mathrm{E} / \mathrm{J} / \mathrm{K}$, can infect chickens [24-27]. Except for the ALV-E subgroup, the other six subgroups are exogenous ALVs, which can cause severe pathogenicity in chickens [28-30]. ALV-E can interfere with the detection of exogenous ALVs because its gene was inserted into the chicken genome early in its evolution, but its pathogenicity is weak $[24,31]$. DF1 cells, a chicken fibroblast cell line with resistance to endogenous ALV [32, 33], have been widely applied to the isolation or detection of exogenous ALVs [34-36]. In this study, we assessed the effect of chicken TRIM25 on the replication of ALV-A in cells.

In this study, DF1 cells were inoculated with the ALVA-SDAU09C1 strain, which was isolated from imported ancestral breeding broiler chickens in 2009 [19] and was reported to replicate in DF1 cells and to be pathogenic in chickens $[19,20,37]$. The expression of chicken TRIM25 at both the mRNA and protein levels was significantly increased, which suggests that the antiviral responses mediated by TRIM25 were induced. To further confirm this finding, the ALV-A-SDAU09C1 strain was inoculated into 1-day-old SPF chickens, and the relative 
expression of TRIM25 in the spleen, liver, bursa, lung and kidney was detected. The results showed that the expression of TRIM25 mRNA in these tissues was significantly upregulated after ALV-A infection, especially in the spleen, liver and bursa. In the past, it has been confirmed that TRIM25 is expressed in almost all chicken tissues and organs and is expressed at relatively high levels in immune organs, such as the spleen, thymus, and bursa; moreover, TRIM25 can be clearly upregulated 2 days after infection with Newcastle disease virus (NDV) [14, 15], suggesting that TRIM25 might participate in some antiviral responses in these organs as an important component. In addition to that in immune organs, the expression of TRIM25 in the liver and kidney was also dramatically upregulated, which might result from the tissue tropism of ALV-A because high viral loads and more pathological lesions, such as tumours in these tissues after ALV-A infection, were reported in previous studies $[19,20]$.

To analyse the effects of chicken TRIM25 on the replication of ALV-A, the complete gene with a size of $1881 \mathrm{bp}$, which contained an open reading frame (ORF), was amplified from Hy-Line egg-type chicken spleens. The bioinformatics analysis results suggest that the amplified chicken TRIM25 gene has $99.6 \%$ homology with the sequences published by Feng (accession No. NM_001318458.1) and contains the typical functional structural domains, such as a RING domain, two B-box domains, a coiled-coil domain and a SPRY domain (unpublished data). Then, this gene was recombined with a eukaryotic expression plasmid containing the green fluorescent protein (GFP) gene to form a new recombinant overexpression plasmid (TRIM25-GFP); meanwhile, a small interfering RNA (TRIM25-siRNA) was prepared to inhibit the expression of endogenous TRIM25 in DF1 cells. The data show that overexpression of TRIM25 can significantly inhibit the replication of ALV-A; in contrast, knockdown of TRIM25 can significantly promote the replication of ALV-A, confirming that chicken TRIM25 has an inhibitory effect on ALV-A replication.

In some previous studies in mammals, TRIM25 could activate the RIG-I protein, which is a receptor that recognizes viral RNA, and induce the production of type I interferons, such as IFN- $\alpha / \beta$, which can inhibit the replication of some viruses in cells [2,38]. Additionally, it was reported that TRIM25 also plays a role in early innate immunity, such as the activation of the MDA5 mitochondrial antiviral signal protein-TRAF6 antiviral axis and the regulation of p53 level and activity [10], and the MDA5 receptor can interact with $\mathrm{V}$ proteins of a wide variety of paramyxoviruses to block IFN signal transduction pathways [39]; the inhibition of IFN induction is not limited to species, and the inhibition of MDA5 function is not limited to mammalian cells either [40]. However, there are no reports regarding the mechanism by which chicken TRIM25 inhibits viral replication in chickens due to the absence of the RIG-I gene in chickens. In 2015, Cheng et al. reported that the MDA5 protein, which is another viral RNA recognition receptor, can mediate the type I interferon responses induced by NDV, AIV or poly (I:C) in the absence of the RIG-I receptor [18]. In this study, the MDA5 receptor-mediated type I interferon response was assessed in cells in which chicken TRIM25 was overexpressed or downregulated, and the results showed that all the expression levels of MDA5, MAVS and IFN- $\beta$ were dramatically upregulated by the overexpression of chicken TRIM25 in response to both incubation with poly(I:C) and inoculation with ALV-A; in contrast, these levels were significantly downregulated by the knockdown of chicken TRIM25. It can be deduced that chicken TRIM25 can increase MDA5-mediated type I interferon responses to produce antiviral factors, such as IFN- $\alpha / \beta$, to inhibit ALV-A replication. Notably, further research is needed regarding how chicken TRIM25 activates the MDA5-mediated type I interferon response.

In summary, in ALV-A-infected DF1 cells and ALV-Ainfected chickens, the expression of chicken TRIM25 was significantly upregulated, and its overexpression inhibited the replication of ALV-A and upregulated the expression of MDA5, MAVS and IFN- $\beta$. In contrast, knockdown of TRIM25 promoted the replication of ALV-A and downregulated the expression of MDA5, MAVS and IFN- $\beta$. These findings can help improve the understanding of the antiviral activities of chicken TRIM25 and greatly enrich the knowledge of antiviral responses in chickens.

\section{Acknowledgements}

We thank Prof. Zhizhong Cui for his support and kind donation in this study.

\section{Authors' contributions}

$\mathrm{ZJ}, \mathrm{LJ}$ and LH performed the experiments, analysed the data, and drafted the manuscript. GH participated in the design, coordination of the study and revision of the manuscript. ZY and HY conducted part of the experiments. CZ provided some suggestions. All authors read and approved the final manuscript.

\section{Funding}

This study was funded by the Project of Shandong Provincial Natural Science Foundation (ZR2019MC044), the Key Research and Development Program of Shandong Province (Important Science and Technology Innovation Project) (2019JZZY010735), the National Key Research and Development Program of China (2016YFD0500800) and the Funds of Shandong "Double Tops" Program.

\section{Availability of data and materials}

All data generated or analysed during this study are included in this published article.

\section{Ethics approval and consent to participate}

This experiment and all the animal materials in this study were approved by the Animal Ethics Committee of Shandong Animal Protection and Welfare Institute (number: SDAU-2018-28), and all procedures related to the animals and their care conformed to the internationally accepted principles as found in the Guidelines for Keeping Experimental Animals issued by the government. 


\section{Consent to publish}

All the authors agree to the terms of the BioMed Central Copyright and License Agreement and approve publication of this paper in the Journal of Veterinary Research.

\section{Competing interests}

The authors declare that they have no competing interests.

\section{Author details}

${ }^{1}$ Shandong Provincial Key Laboratory of Animal Biotechnology and Disease Control and Prevention, Tai'an 271018, China. ${ }^{2}$ College of Animal Science and Veterinary Medicine, Shandong Agricultural University, Tai'an 271018, China.

\section{Received: 2 July 2020 Accepted: 19 November 2020}

Published online: 09 December 2020

\section{References}

1. Meroni G, Diez-Roux G (2005) TRIM/RBCC, a novel class of 'single protein RING finger'E3 ubiquitin ligases. BioEssays 27:1147-1157

2. Gack MU, Shin YC, Joo CH, Urano T, Liang CY, Sun LJ, Takeuchi O, Akira SZ, Chen ZJ, Inoue S, Jung JU (2007) TRIM25 RING-finger E3 ubiquitin ligase is essential for RIG-I-mediated antiviral activity. Nature 446:916-920

3. Gack MU, Kirchhofer A, Shin YC, Inn KS, Liang C, Cui S, Myong S, Ha T, Hopfner KP, Jung JU (2008) Roles of RIG-I N-terminal tandem CARD and splice variant in TRIM25-mediated antiviral signal transduction. PNAS USA 105:16743-16748

4. Akshay A, Nadia J, Jessica J (2013) Crystal structure of the TRIM25 B30.2 (PRYSPRY) domain: a key component of antiviral signaling. Biochem Soc 456:231-240

5. Inoue S, Orimo A, Hosoi T, Kondo S, Toyoshima H, Kondo T, Ikegami A, Ouchi Y, Orimo H, Muramatsu M (1993) Genomic binding-site cloning reveals an estrogen-responsive gene that encodes a RING finger protein. PNAS USA 90:11117-11121

6. Ozato K, Shin DM, Chang TH, Morse HC (2008) Trim family proteins and their emerging roles in innate immunity. Nat Rev Immunol 8:849-860

7. Takeuchi O, Akira S (2008) MDA5/RIG-I and virus recognition. Curr Opin Immunol 20:17-22

8. Rajsbaum R, García-Sastre A, Versteeg GA (2014) TRIMmunity: the roles of the TRIM E3-ubiquitin ligase family in innate antiviral immunity. J Mol Biol 426:1265-1284

9. Urano T, Saito T, Tsukui T, Fujita M, Hosoi T (2002) Efp targets 14-3-3 sigma for proteolysis and promotes breast tumour growth. Nature 417:871-875

10. Martín-Vicente M, Medrano LM, Resino S, García-Sastre A, Martínez I (2017) TRIM25 in the regulation of the antiviral innate immunity. Front Immunol 8:1187

11. Kang DC, Gopalkrishnan RV, Wu Q, Jankowsky E, Pyle AM, Fisher PB (2002) mda-5: an interferon-inducible putative RNA helicase with doublestranded RNA-dependent ATPase activity and melanoma growth-suppressive properties. PNAS USA 99:637-642

12. Wei YN, Zhou H, Wang AQ, Sun LP, Wang MS, Jia RY, Zhu DK, Liu MF, Yang Q, Wu Y, Sun KF, Chen XY, Cheng AC, Chen S (2016) Trim25 identification in the chinese goose: gene structure, tissue expression profiles, and antiviral immune responses in vivo and in vitro. Biomed Res Int 2016:1-14

13. Jin Y, Jia K, Zhang W, Xiang Y, Yi M (2019) Zebrafish trim 25 promotes innate immune response to RGNNV infection by targeting 2card and rd regions of RIG-I for k63-linked ubiquitination. Front Immunol 10:2805

14. Feng ZQ, Cheng Y, Yang HL, Zhu Q, Yu DD, Liu YP (2015) Molecular characterization, tissue distribution and expression analysis of TRIM25 in Gallus gallus domesticus. Gene 561:138-147

15. Chu Y, Ma WJ, Hu WG, Chen R, Qiu JH, Guo HJ, Li HM (2018) Immunohistochemistry assay for detecting chicken TRIM25 in different tissues. Acta Veterinaria et Zootechica Sinica 49:865-870

16. Zou J, Chang M, Nie P (2009) Secombes. Origin and evolution of the RIG-I like RNA helicase gene family. BMC Evol Biol 9:85

17. Barber MR, Aldridge JR, Webster RG, Magor KE (2010) Association of RIG-I with innate immunity of ducks to influenza. PNAS USA 107:5913-5918
18. Cheng YQ, Sun YJ, Wang HG, Yan YX, Ding C, Sun JH (2015) Chicken STING mediates activation of the IFN gene independently of the RIG-I gene. J Immunol 195:3922-3936

19. Zhang QC, Zhao DM, Guo HJ, Cui ZZ (2010) Isolation and identification of a subgroup A avian leukosis virus from imported meat-type grandparent chickens. Virol Sin 25:130-136

20. Zhang ZS, Hu WG, Li BQ, Chen R, Guo HJ, Li HM (2019) Comparison of viremia, cloacal virus shedding, antibody responses and pathological lesions in adult chickens, quails, and pigeons infected with ALV-A. Sci Rep 9:3027

21. Chen H, Wang YX, Zhao P, Li JL, Cui ZZ (2012) Acute Fibrosarcomas caused by avian leukosis virus subgroup J associated with $v$-fps oncogene. J Anim Vet Adv 11:2910-2916

22. Wu XP, Zhao JR, Zeng YK, Wu YJ, Wang QX, Wu BC, Huang YF (2017) A novel avian retrovirus associated with lymphocytoma isolated from a local Chinese flock induced significantly reduced growth and immune suppression in SPF chickens. Vet Microbiol 205:34-39

23. Meng FF, Li QC, Zhang YB, Cui ZZ, Chang S, Zhao P (2018) Isolation and characterization of subgroup J Avian Leukosis virus associated with hemangioma in commercial Hy-Line chickens. Poult Sci 97:2667-2674

24. Fadly A (2003) Leukosis/sarcoma group. In: Saif YM (ed) Diseases of poultry, 11th edn. Iowa State University Press, Ames, pp 465-516

25. Meng FF, Dong X, Chang S, Zhao P, Cui ZZ (2016) A deep sequencing reveals significant diversity among dominant variants and evolutionary dynamics of avian leukosis viruses in two infectious ecosystems. BMC Vet Res 12:287

26. Li Y, Fu JY, Cui S, Meng FF, Cui ZZ, Chang S, Zhao P (2017) Gp85 genetic diversity of avian leukosis virus subgroup J among different individual chickens from a native flock. Poultry Sci 96:1100-1107

27. Su Q, Li Y, Li WH, Cui S, Tian SB, Cui ZZ, Zhao P, Chang S (2018) Molecular characteristics of avian leukosis viruses isolated from indigenous chicken breeds in China. Poultry Sci 97:2917-3292

28. Zhao P, Cui ZZ, Ma CT, Dong X, Li DQ (2012) Serological survey of the avain leukosis virus infection in China native chickens. J Anim Vet Adv 11:2584-2587

29. Li D, Qin L, Gao H, Yang B, Liu W, Qi X, Wang Y, Zeng X, Liu S, Wang X, Gao Y (2013) Avian leukosis virus subgroup A and B infection in wild birds of Northeast China. Vet Microbiol 163:257-263

30. Dong X, Ju SD, Chen JX, Meng FF, Sun P, Li Y, Wang X, Wang YX, Chang S, Zhao P, Cui ZZ (2016) Karyotype analysis of the acute fibrosarcoma from chickens infected with subgroup J avian leukosis virus associated with v-src oncogene. Avian Pathol 45:202-207

31. Qu Y, Sun H, Sun M, Li D, Qin L, Gao Y, Wang X, Liu S (2012) Sequence analysis for the complete proviral genome of avian leukosis virus subgroup j associated with haemangiomas, leiomyosarcomas and myelomas in layer flocks. Acta Vet Hung 60:343-354

32. Himly M, Foster DN, Bottoli I, lacovoni JS, Vogt PK (1998) The DF-1 chicken fibroblast cell line: transformation induced by diverse oncogenes and cell death resulting from infection by avian leukosis viruses. Virology 248:295-304

33. Schaefer-Klein J, Givol I, Barsov EV, Whitcomb JM, VanBrocklin M, Foster DN, Federspiel MJ, Hughes SH (1998) The EV-0-derived cell line DF-1 supports the efficient replication of avian leukosis-sarcoma viruses and vectors. Virology 248:305-311

34. Bagust TJ, Fenton SP, Reddy MR (2004) Detection of subgroup J avian leukosis virus infection in Australian meat-type chickens. Aust Vet J 82:701-706

35. Wang $X Z$, Wang $B$, Zhang PP, Cheng HG, Sun SH (2013) The passage of cells can improve the detection rate of avian leukosis virus to facilitate the elimination of avian leukosis in chickens. SpringerPlus 2:138. https ://doi.org/10.1186/2193-1801-2-138

36. Qu YJ, Niu YJ, Li N, Sun W, Lv CW, Wang PF, Zhang GH, Liu SD (2016) Viral proliferation and expression of tumor-related gene in different chicken embryo fibroblasts infected with different tumorigenic phenotypes of avian leukosis virus subgroup. J Poultry Sci 95:2383-2390

37. Zhang D, Li H, Zhang Z, Sun S, Cheng Z, Liu J, Zhao P, Ren Q, Guo H (2015) Antibody responses induced by recombinant ALV-A gp85 protein vaccine combining with CpG-ODN adjuvant in breeder hens and the protection for their offspring against early infection. Antiviral Res 116:20-26 
38. Sanchez JG, Chiang JJ, Sparrer KM, Alam SL, Chi M, Roganowicz MD, Sankaran B, Gack MU, Pornillos O (2016) Mechanism of TRIM25 catalytic activation in the antiviral RIG-I pathway. Cell Rep 16:1315-1325

39. Andrejeva J, Childs KS, Young DF, Carlos TS, Stock N, Goodbourn S, Randall RE (2004) The $V$ proteins of paramyxoviruses bind the IFN inducible RNA helicase, mda-5, and inhibit its activation of the IFN-beta promoter PNAS USA 101:17264-17269

40. Childs K, Stock N, Ross C, Andrejeva J, Hilton L, Skinner M, Randall R, Goodboum S (2007) Mda-5, but not RIG-1, is a common target for paramyxovirus $V$ proteins. Virology 359:190-200

\section{Publisher's Note}

Springer Nature remains neutral with regard to jurisdictional claims in published maps and institutional affiliations.
Ready to submit your research? Choose BMC and benefit from:

- fast, convenient online submission

- thorough peer review by experienced researchers in your field

- rapid publication on acceptance

- support for research data, including large and complex data types

- gold Open Access which fosters wider collaboration and increased citations

- maximum visibility for your research: over 100M website views per year

At BMC, research is always in progress.

Learn more biomedcentral.com/submissions 